\title{
Potential-energy surfaces for charge exchange between singly charged ions and a LiF surface
}

\author{
Ludger Wirtz* and Joachim Burgdörfer \\ Institute for Theoretical Physics, Vienna University of Technology, Wiedner Hauptstrasse 8-10/136, A-1040 Wien, Austria \\ Michal Dallos, Thomas Müller, and Hans Lischka \\ Institute for Theoretical Chemistry and Structural Biology, Vienna University, Währinger Strasse 17, A-1090 Wien, Austria
}

(Received 31 May 2003; published 23 September 2003)

\begin{abstract}
We analyze the adiabatic potential-energy surfaces relevant for neutralization of singly charged ions in slow vertical incidence onto a lithium fluoride surface. The surface is represented by a cluster of varying size augmented by point charges of alternating sign in order to include the proper Madelung potential of the ionic crystal. Our calculation proceeds on the multiconfiguration self-consistent-field and multireference configuration-interaction levels. Size-consistency corrections based on the Davidson correction and multireference averaged quadratic coupled cluster methods are included as well. We emphasize the importance of a proper treatment of electron correlation signifying the polarization of the surrounding cluster environment in $a b$ initio calculations of charge transfer at surfaces. From the topology of the surfaces, in particular the existence or absence of avoided crossings (or, more generally, conical intersections), qualitative predictions for the neutralization process can be made. The comparative analysis of potential curves for $\mathrm{H}^{+}, \mathrm{C}^{+}, \mathrm{S}^{+}$, and $\mathrm{Ne}^{+}$ projectiles provides an explanation for the recently observed threshold behavior for potential sputtering.
\end{abstract}

DOI: $10.1103 /$ PhysRevA.68.032902

PACS number(s): 34.50.Dy, 79.20.Rf, 82.30.Fi

\section{INTRODUCTION}

In spite of a wealth of experimental data on the interaction between ion beams and surfaces and concomitant theoretical efforts [1], an accurate $a b$ initio description of charge transfer during ion-surface collisions has yet to be achieved. The challenge originates in the many-body (both many-electron and many-nuclei) character of the problem on one hand and the dynamical nature of such a collision process involving a multitude of excitations of the many-body system far from the ground state on the other. Moreover, the strong perturbation by the localized Coulomb field of the incident ion breaks most, if not all, symmetries of the crystal field and renders Bloch functions meaningless as starting point for chargetransfer calculations. Instead, the localized charge exchange between the projectile ion and surface atoms (ions) calls for methods employed in the field of ion-atom and ion-molecule collisions [2-4].

The interaction between ions and metal surfaces seems to be more easily accessible by $a b$ initio methods than the interaction between ions and insulator surfaces. Metals are frequently described in the jellium approximation where the conduction-band electrons are subject to the homogeneous potential of the smeared-out positive atom-core charges. At the surface, this leads to an electron density that varies perpendicular to the surface plane but is constant parallel to the surface. The presence of the projectile ion in front of the surface breaks this two-dimensional translational symmetry but still preserves the cylindrical symmetry of the system. As a first step towards the solution of the corresponding twodimensional time-dependent Schödinger equation, atomic

\footnotetext{
*Present address: Department of Material Physics, University of the Basque Country and Donostia International Physics Center, Po. Manuel de Lardizabal 4, 20018 Donostia-San Sebastián, Spain.
}

resonances, i.e., the position and the width of energy levels, in front of jellium surfaces have been calculated with high accuracy [5]. With increasing computer power, a dynamical treatment of this system with time-dependent densityfunctional theory seems to become feasible [6]. For an ion in front of an ionic crystal surface such as $\mathrm{LiF}, \mathrm{NaCl}$, or $\mathrm{MgO}$, also the cylindrical symmetry is broken, requiring - in principle - the solution of a time-dependent many-body Schrödinger equation for an extended system in three dimensions. Since this has so far not been computationally feasible, only a single surface atom or a small number of surface atoms is usually described $a b$ initio and surrounded by an array of point charges to represent the residual crystal.

The use of surface and bulk embedded clusters has a long tradition in quantum chemistry calculations of local perturbations in an infinitely extended system. For example, calculations have been performed for the spectra of color centers in alkali halides [7], the spectra of impurities in a crystalline environment [8], surface and bulk excitons in LiF [9], adsorption energies at a $\mathrm{MgO}$ surface [10], and energies of surface and defect states in $\mathrm{MgO}$ [11]. For charge exchange between ions and alkali-halide surfaces, Souda et al. [12] determined energy-level diagrams of molecular orbitals on the Hartree-Fock level for the interaction of $\mathrm{H}^{+}$with embedded $\mathrm{Li}^{+} \mathrm{Cl}_{5}{ }^{-}$and $\mathrm{Li}_{5}{ }^{+} \mathrm{Cl}^{-}$clusters. García et al. [13] investigated the negative-ion conversion of $\mathrm{H}^{+}$during large-angle scattering at a $\mathrm{LiF}$ surface in the framework of a timedependent Anderson Hamiltonian [14]. They calculated Hartree-Fock wave functions of embedded $\mathrm{Li}_{5}{ }^{+} \mathrm{F}^{-}$and $\mathrm{Li}^{+} \mathrm{F}_{5}{ }^{-}$clusters as input for the matrix elements of the model Hamiltonian. Zeijlmans van Emmichoven et al. [15] discussed energy-level diagrams of molecular orbitals for the system $\mathrm{H}+\mathrm{F}^{-}$in a crystalline environment from which they constructed diabatic and adiabatic potential-energy curves for negative-ion conversion followed by emission of an electron during grazing-incidence scattering of protons at a $\mathrm{LiF}$ 
surface. Borisov et al. [16] performed Hartree-Fock calculations for the negative-ion conversion of $\mathrm{F}^{0}$ during grazing incidence with $\mathrm{LiF}$ and $\mathrm{MgO}$ surfaces. They used a small embedded surface cluster with a single negative ion as electron donor and constructed diabatic energy curves and coupling-matrix elements from calculations of atomic states.

In this paper, we critically revisit the embedded-cluster approach for ion-surface scattering. The point of departure of our analysis is the observation that on the Hartree-Fock level, effects of correlation and polarization resulting from transferring an electron from the surface to the projectile are missing. This difficulty has profound consequences for charge-transfer processes. The lack of correlation effects does not only result in quantitative inaccuracies of the potential-energy curves, but may prevent to even qualitatively predict the correct topology of the energy surfaces. Specifically, for some ion-surface collision systems, HF calculations lead to a wrong ordering of the levels and become meaningless as input for dynamical charge-transfer calculations. We therefore apply the multiconfiguration selfconsistent-field (MCSCF) method [17] and the multireference configuration-interaction method with single and double excitations (MR-CISD) [18]. Furthermore, we use the extended Davidson correction $[19,20]$ and the MR-AQCC (multireference average quadratic coupled cluster) method [21] to assess size-consistency errors [22] present in the MRCISD calculations. Within this framework we are in the position to determine the potential surfaces for the combined system of a singly charged ion incident on a LiF surface.

One application of our calculations is the determination of the recently observed "threshold behavior" for potential sputtering of LiF by slow singly charged ions [23]. Potential sputtering, i.e., sputtering due to the recombination energy or the potential energy that the projectile carries into the collision, was observed for all ions with a recombination energy higher than $10 \mathrm{eV}$. In the model of defect-mediated sputtering [24], the ablation of surface particles is explained by the creation of a hole by electron transfer to the projectile and the subsequent removal of a loosely bound $\mathrm{F}^{0}$ from the surface. Therefore, the threshold for potential sputtering is directly related to the energetic threshold for charge exchange. Employing simple estimates for asymptotic potential curves and extrapolating to smaller distance, a threshold value of 10 $\mathrm{eV}$ was inferred [25]. The present calculation provides a quantitative underpinning of this threshold behavior. The key point is that for the determination of the threshold value of the recombination energy, the accurate knowledge of the energy surface suffices. The full solution of the nonadiabatic transition matrix, which will be the subject of a future investigation, is not required for this purpose. In Sec. II we briefly review the methods underlying our calculations. In Sec. III we present results of embedded cluster calculations for the unperturbed surface at different levels of approximation in order to gauge their reliability. In Sec. IV we present results for the energy surfaces for different ions impinging on small embedded clusters. Effects of cluster size are studied in Sec. $\mathrm{V}$ where we consider protons $\left(\mathrm{H}^{+}\right)$incident on large clusters at different levels of approximation. The paper concludes with a summary and outlook.

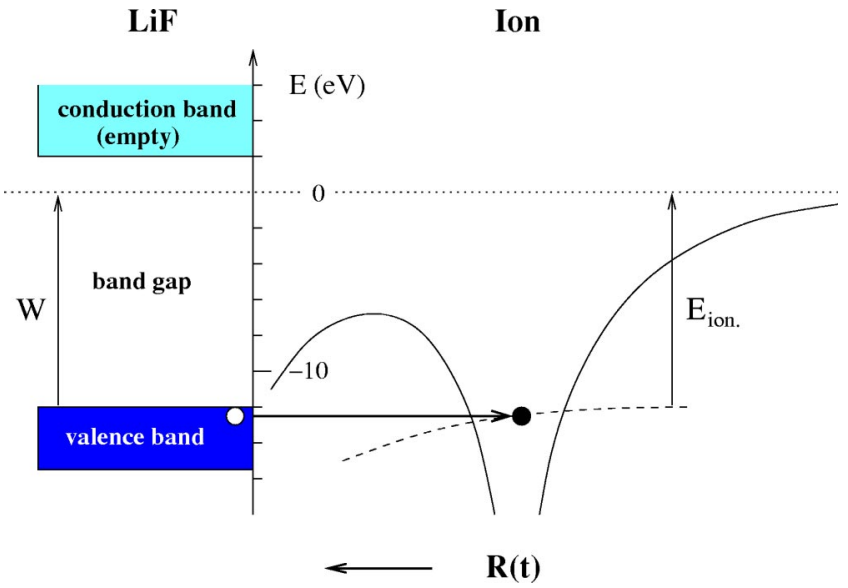

FIG. 1. Orbital energy picture for the charge exchange between projectile ion and surface: schematic picture of band structure of $\mathrm{LiF}$ and of the capture level in the Coulombic potential of the ion core.

\section{METHOD OF CALCULATION}

Charge exchange between ions and surfaces is frequently discussed within the framework of orbital energies as shown in Fig. 1. The left-hand side of the figure displays the band structure of $\mathrm{LiF}$ which is a prototype of an insulator with a wide band gap. The valence-band edge (corresponding to the negative work function) lies at about $-W=-12.3 \mathrm{eV}$ [26] and the bandwidth is about $\Gamma=3.4 \mathrm{eV}$ [27]. The energy of the center of the band can be roughly estimated $[28,16]$ as the sum of the (negative) electron affinity, $-E_{a}=-3.4 \mathrm{eV}$ and the Madelung potential at the site of a surface fluorine which-assuming fractional charges of \pm 0.86 following Ref. [26] —amounts to about $V_{M}=-11.3 \mathrm{eV}$ [29]. In the embedded-cluster approach, the surface is represented by a finite cluster of $\mathrm{Li}^{+}$and $\mathrm{F}^{-}$ions embedded into an array of positive and negative point charges which simulates the residual, infinitely extended, crystal [see Fig. 5(g) below]. The "valence band" of the embedded cluster is not continuous but consists of a discrete set of levels that arise from the energy splitting due to the overlap of the $2 p$ atomic orbitals of the fluorine ions. For large clusters, the set remains discrete but becomes dense and its width converges towards the bandwidth of the infinite system (see Sec. III). However, the position of the "band" as given by the orbital energies of the $\mathrm{F}_{2 p}$-like orbitals is too low by about $3 \mathrm{eV}$. This is a wellknown deficiency of Hartree-Fock band-structure calculations [30,31] and will be the topic of Sec. III.

The right-hand side of Fig. 1 displays the energy level (capture level) of an outer electron in the Coulombic potential of the projectile ion. During the approach of the projectile towards the surface, the capture level is shifted due to the surface potential, the dielectric response of the surface to the presence of the Coulomb field of the ion, and the interaction with the hole left in the surface after transferring an electron to the projectile $[25,28]$. At the same time, the energy of the locally distorted band is shifted downwards due to the attractive Coulomb potential of the positive-ion core. Resonant charge transfer between the valence band of the surface and 
the capture level of the projectile can take place when the energy of the capture level matches the energy of the band [32]. Therefore, in the picture of orbital energies, an approximate determination of the threshold for charge transfer relies on estimates whether or not the capture level dives into the valence band as the ion approaches the surface [25].

At small distances, the strong local perturbation of the valence band leads to the formation of quasimolecular orbitals encompassing both surface atoms and the projectile. This observation suggests the use of the methodology for the treatment of slow ion-atom collisions. The first step in the description of a slow ion-atom collision for the reaction

$$
A^{+}+B \rightarrow A+B^{+},
$$

where $A^{+}$denotes the projectile ion and $B$ the target atom, is the calculation of energies and wave functions for the ground state of the system and for one or several excited states corresponding to the configurations $A^{+}+B$ and $A+B^{+}$, respectively. The energies $E_{i}(\vec{R})$ and wave functions $\psi_{i}(\vec{r} ; \vec{R})$ depend parametrically on the internuclear separation $\vec{R}$. These "adiabatic" electronic states at fixed distance are used as a basis for the expansion of the time-dependent wave function $\Psi(\vec{r}, \vec{R}(t), t)$, which is the solution of the time-dependent Schrödinger equation (TDSE)

$$
i \dot{\vec{R}} \vec{\nabla}_{R} \Psi(\vec{r}, \vec{R}(t), t)=\hat{H}(\vec{r}, \vec{R}(t)) \Psi(\vec{r}, \vec{R}(t), t)
$$

when the internuclear coordinate becomes time dependent. Thereby, the different adiabatic states couple through the matrix elements $\left\langle\psi_{i}(\vec{R})\left|\vec{\nabla}_{R}\right| \psi_{j}(\vec{R})\right\rangle$ [33]. The coupling is strongly peaked near avoided crossings or conical intersections which effectively control the nonadiabatic transitions.

In the simplest case, only two adiabatic energy curves with eigenstates $\psi_{1}(R)$ and $\psi_{2}(R)$ need to be calculated for an adequate description of charge exchange. The identification of avoided crossings in the potential-energy curves allows a qualitative (and sometimes quantitative) estimate when the "diabatic" level curves can be locally approximated either by intersecting linear curves (Landau-Zener model [34]) or by closely spaced levels running in parallel for an extended interval of $R$ (Rosen-Zener-Demkov model [35]). Of course, a detailed quantitative analysis requires the solution of the TDSE within a given subspace with accurate input for potential curves and coupling-matrix elements.

The accurate calculation of excited states and couplingmatrix elements is already a considerable challenge for effective one-electron systems. In many-electron systems this problem is even more difficult. The most promising, however computationally demanding, way seems to be the use of a MR-CI approach for the calculation of wave functions and a finite-difference method to calculate the coupling-matrix elements [36]. In this paper we focus on the calculation of the adiabatic potential surfaces within the framework of the MR-CI approach. The analysis of coupling-matrix elements is deferred to a future study. In the present case, $A$ in Eq. (2.1) refers to the projectile while $B$ refers now to the entire embedded surface cluster representing the local environment

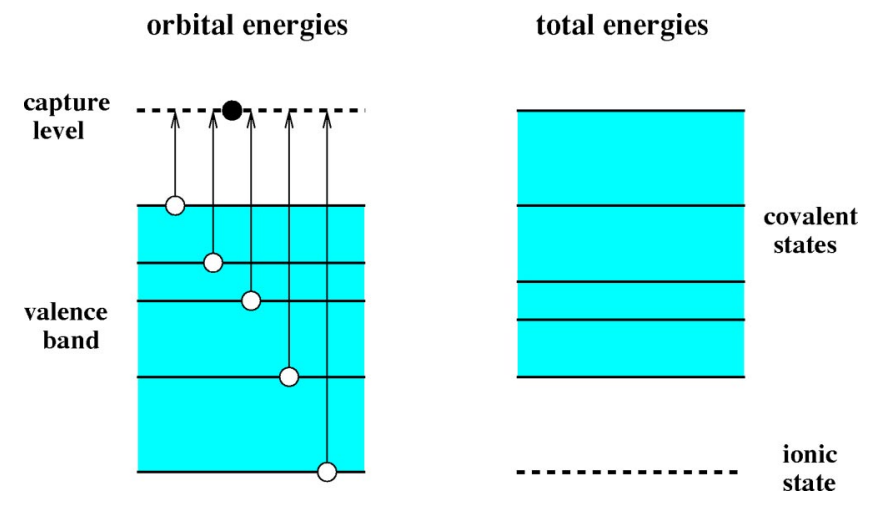

FIG. 2. Comparison of orbital energy and total-energy picture.

of the surface near the region of impact. Since the valence band is represented by a discrete set of $n$ states from any of which an electron can be transferred to the projectile, a total of $n+1$ potential-energy surfaces is required. One of these is an ionic state (charged projectile plus neutral surface, $A^{+}$ $+B)$ and $n$ states correspond to "covalent" states where the projectile is neutral with a hole left behind in the surface $\left(A+B^{+}\right)$. Figure 2 illustrates the correspondence between the orbital energy picture of Fig. 1 and the total-energy potential surfaces calculated in the present work. For manyelectron systems with strong correlation effects the latter represent the more appropriate framework. When comparing the following calculations with earlier work in this field, it should be kept in mind that the total-energy picture displays a reverse ordering of levels compared to the orbital energy picture. For example, in the case of Fig. 2, where the capture level is higher than the upper valence-band edge, the ionic state is the lowest one and all the covalent states representing a hole in the valence band lie higher in energy.

For our calculations, we employ the quantum chemistry code COLUMBUS [37-39] the specialization of which is the implementation of multireference, multiconfiguration methods. For the calculation of atomic-orbital integrals the program system DALTON [40] was used. The calculations usually proceed in three stages. As a first step, we calculate the ground state of the system on the Hartree-Fock or selfconsistent-field (SCF) level in order to obtain starting orbitals for the following two steps. Clearly, a single configuration is not sufficient to represent excited states. Therefore, in the second step, we use a multiconfiguration SCF (MCSCF) method [17] in order to obtain approximate energies and orthonormalized wave functions for the ground and the excited states. The active space that defines the number of configurations comprises the valence orbital(s) of the projectile and the $\mathrm{F}_{2 p}$ orbitals of the surface cluster. The third step is a MR-CI method [18] with single and double excitations (MRCISD). All the configurations of the MCSCF wave function are taken as reference configurations for which single and double excitations are allowed.

The MR-CISD method contains dynamic electron correlation effects and represents an important step beyond the MCSCF approach. However, it lacks size consistency, i.e., the MR-CISD energy does not scale linearly with the number of particles (see, e.g., Ref. [22] and further references 


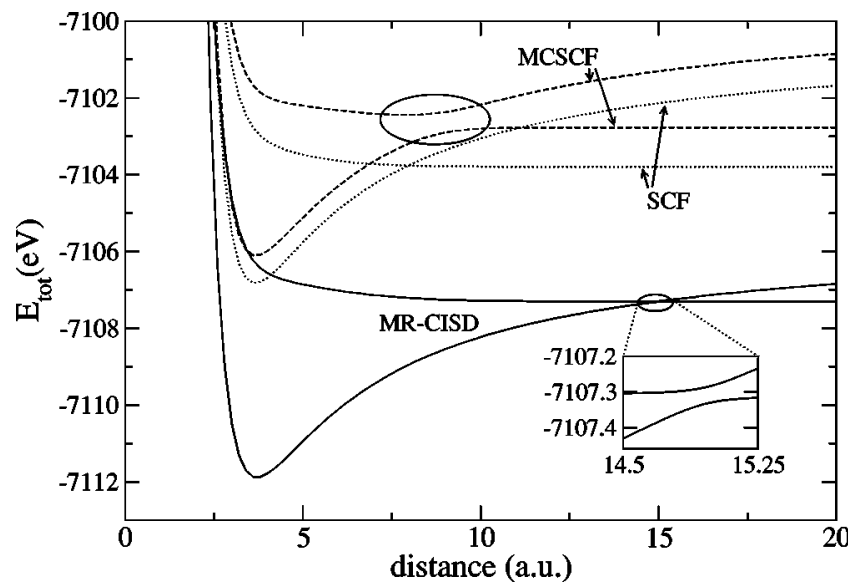

FIG. 3. Diatomic quasimolecular potential-energy curves for $\mathrm{Na}^{0}+\mathrm{F}^{0}$ and $\mathrm{Na}^{+}+\mathrm{F}^{-}$on the SCF (dotted), MCSCF (dashed), and MR-CISD (solid) level. Avoided crossings are marked by ellipses. Calculations are performed with a DZ basis set with additional polarization and diffuse functions for fluorine [41,42].

therein) and does not give the correct dissociation limit when a molecule is broken into subsystems. In order to correct for this deficiency, several methods have been developed. The simplest one is the Davidson method [19] including an $a$ posteriori correction due to higher excitations. This method has been extended later for the multireference case [20]. A more consistent way to include size-consistency corrections is the MR-AQCC method [21], which specializes on multireference cases.

Figure 3 illustrates the different levels of accuracy for the example of an alkali-halide molecule in the gas phase. At large distances, the ionic state $\left(\mathrm{Na}^{+}+\mathrm{F}^{-}\right)$lies energetically higher than the covalent state $\left(\mathrm{Na}^{0}+\mathrm{F}^{0}\right)$ because the ionization potential of $\mathrm{Na}$ is larger than the electron affinity of F. At small distances, the level ordering is reversed due to the attractive Coulomb potential between the ions. At intermediate distance, the energy surfaces would intersect except for an avoided crossing between levels of the same symmetry. In the MR-CISD calculation, the electron affinity of fluorine is properly described, which energetically favors the ionic state and shifts the avoided crossing by 5 a.u. towards larger distances relative to the position in the MCSCF calculation. The SCF calculation completely fails to reproduce the avoided crossing at all, since the wave function is expressed by a single Slater determinant and thereby forced into either the ionic or the covalent configuration. The pronounced differences in the NaF total-energy curves on the different levels of approximation indicate that also for collision systems involving $\mathrm{F}^{-}$ions embedded in a surface, a careful calculation of the potential-energy surfaces with high accuracy is indispensable.

For the collision system consisting of projectile and LiF surface, the potential-energy surfaces depend on the coordinates $(x, y)$ parallel to the surface and the distance $z$ of the projectile from the surface. In this paper, we calculate cuts along the $z$ axis through the potential-energy surfaces as illustrated in Fig. 4. These cuts can be used to infer the chargetransfer probability for vertical incidence of the projectile on

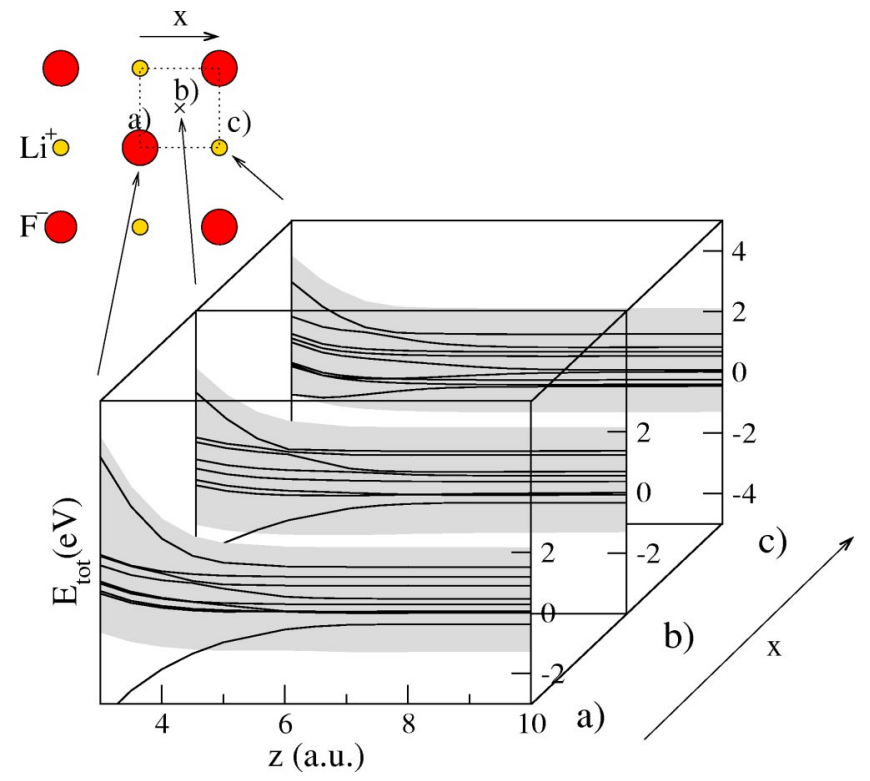

FIG. 4. Schematic plot of the potential-energy surfaces for a $\mathrm{H}^{+}$ ion colliding with a LiF surface. The three cuts along the $z$ axis (perpendicular to the surface) correspond to different positions of vertical impact in the two-dimensional unit cell of the LiF surface (see inset): (a) on top of an $\mathrm{F}^{-}$, (b) at the midpoint between adja-

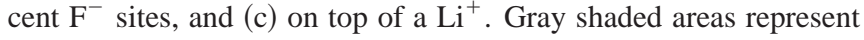
the quasicontinuous band of covalent states. For details see Ref. [43].

different positions in the two-dimensional unit cell of the LiF surface. (For arbitrary collision trajectories, a dense grid of these one-dimensional cuts needs to be calculated to reconstruct the potential surface.) The gray shaded areas represent the quasicontinuous band of covalent states. For each collision geometry, this band is represented by a finite number of discrete states which depends on the size of the embedded cluster used to simulate the surface [43]. The reliability of an embedded-cluster representation for the infinitely extended surface is investigated in the following section.

\section{IONIZATION POTENTIAL AND BANDWIDTH OF EMBEDDED SURFACE CLUSTERS}

An important figure of merit for the reliability of embedded-cluster calculations for charge-exchange calculations is the ionization potential of the embedded cluster in absence of any perturbation by the projectile Coulomb field. In the limit of large clusters, the work function of $\mathrm{LiF}$ should emerge. The importance of the work function is derived from the fact that the relative magnitude of work function and recombination energy of the projectile ion determines the ordering of the band levels and the capture level at large distances (see Fig. 1).

We have performed calculations of the ionization potential of the different embedded clusters shown in Fig. 5. For all clusters, the number of included $\mathrm{Li}^{+}$ions exceeds the number of $\mathrm{F}^{-}$ions, thereby surrounding each active $\mathrm{F}^{-}$ion with active $\mathrm{Li}^{+}$ions on all sides in order to prevent artificial distortion of the electron density. While the active cluster is positively charged, it is surrounded by point charges [see Fig. 
a)

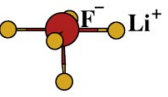

b)
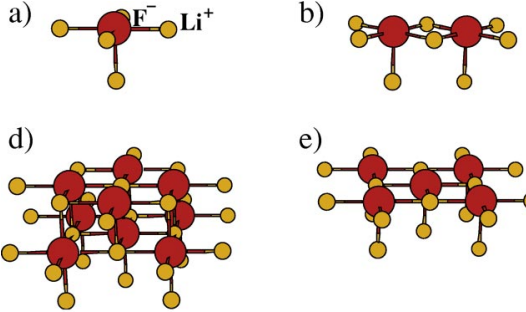

e)

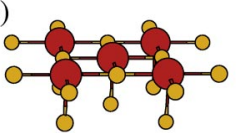

c)
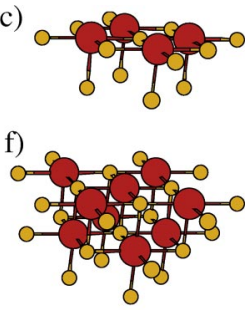

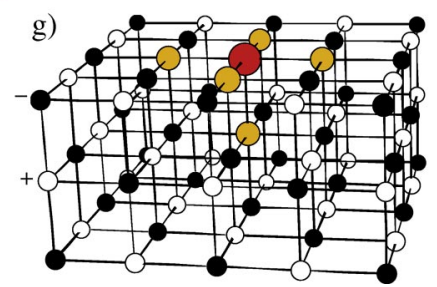

FIG. 5. Different embedded clusters for which ionization potentials are listed in Table I: (a) $\mathrm{Li}_{5}{ }^{+} \mathrm{F}^{-}$, (b) $\mathrm{Li}_{8}{ }^{+} \mathrm{F}_{2}{ }^{-}$, (c) $\mathrm{Li}_{13}{ }^{+} \mathrm{F}_{4}{ }^{-}$, (d) $\mathrm{Li}_{26}{ }^{+} \mathrm{F}_{9}{ }^{-}$, (e) $\mathrm{Li}_{17}{ }^{+} \mathrm{F}_{5}{ }^{-}$, and (f) $\mathrm{Li}_{25}{ }^{+} \mathrm{F}_{9}{ }^{-}$. Panel (g) demonstrates the embedding of cluster (a) in positive and negative point charges.

$5(\mathrm{~g})]$ such that the total system is neutral, provided that the total number of lattice ions is even. If the crystal was charged as in Ref. [9], the ionization potential of the cluster would be shifted. The total number of lattice ions (point charges plus active ions $)$ is $196\left(n_{x} \times n_{y} \times n_{z}=7 \times 7 \times 4\right)$ for clusters (a) and (c)-(f). For cluster (b) we use 256 ions $\left(n_{x} \times n_{y} \times n_{z}\right.$ $=8 \times 8 \times 4)$. In all cases the lattice is sufficiently large as to reproduce the Madelung potential $V_{M}$ with an accuracy of about $10^{-2} \mathrm{eV}$. This error is one order of magnitude smaller than typical errors introduced, e.g., by the choice of a finite basis set for the molecular orbitals.

As basis set, we use the pseudobasis of Stevens et al. [44] for the $\mathrm{F}^{-}$and the $\mathrm{Li}^{+}$ions. In this basis, the $1 \mathrm{~s}$ core electrons are replaced by a pseudopotential. As described in the Appendix, this basis is a compromise between accurately describing a single negative fluorine anion and being able to describe a larger cluster of ions. For the $\mathrm{Li}^{+}$ions, we truncate the two $p$-type Gaussian functions from the basis. Since on the SCF level, the admixture of $\mathrm{Li} 2 p$ orbitals to the valence band of LiF is very small, this introduces only a minor difference of the orbital energies as compared to a calculation with the full pseudobasis; and since the number of $\mathrm{Li}^{+}$ions greatly exceeds the number of fluorines, this

truncation facilitates the calculation of larger clusters, up to $\mathrm{Li}_{26}{ }^{+} \mathrm{F}_{9}{ }^{-}$, even on the MR-CI level. We note that for a highly accurate calculation of correlation energy, electronic excitations from the valence-band orbitals to the $\mathrm{Li}_{2 p}$ orbitals as well as to higher orbitals of the fluorines would be desirable. However, this would exceed the current limitations of the code with respect to the number of allowed basis functions. Our intention here is to systematically investigate the influence of the cluster form and cluster size on the ionization potential. Therefore, we have chosen the same basis set of modest size for all clusters.

In Table I we compare the results for the clusters depicted in Fig. 5 as well as for a single active fluorine embedded into point charges as used in Ref. [16]. Note that cluster (d) results from adding an additional layer of active fluorines below the surface to cluster (c). In the same way cluster (f) results from cluster (e). The simplest estimate of the ionization potential is (via Koopmans' theorem) the orbital energy of the highest occupied molecular orbital (HOMO) in the SCF calculation of the neutral cluster, $\left|\epsilon_{\text {HомO }}\right|$, given in the first row of Table I. More reliable is the calculation of the ionization potential as the total-energy difference between the neutral and the positively charged cluster, which has been performed on different levels of accuracy, i.e., on the MCSCF and the MR-CISD levels. We also calculated the extended Davidson correction to the $\mathrm{CI}$ and performed a MR-AQCC calculation. All calculations are performed for configurations with $A_{1}$ symmetry within the $C_{2 v}$ point-group symmetry.

The active space of the MCSCF contains all $\mathrm{F}_{2 p}$-like orbitals which have $A_{1}$ symmetry. For the neutral cluster which has a closed-shell structure, all $\mathrm{F}_{2 p}$ orbitals are filled with electrons. In this case, the active space comprises just one configuration and the MCSCF method coincides with the SCF method. Upon ionizing the cluster, an electron hole is created in one of the $\mathrm{F}_{2 p}$-like molecular orbitals. For example, for the $\mathrm{Li}_{25}{ }^{+} \mathrm{F}_{9}{ }^{-}$cluster, eight molecular orbitals of $A_{1}$ symmetry can be formed. The ground state is a superposition of different configurations and cannot be forced into the form of a single Slater determinant (which is why the SCF method does not converge in this case). The MCSCF method allows for the solution of the ground and several excited states at the same time by way of state averaging. We perform a calculation for as many states as there are hole configurations of $A_{1}$ symmetry. Since in a state-averaged cal-

TABLE I. Ionization potential $E_{\text {ion }}$ calculated on different levels of approximation and band width $\Gamma$ of the finite embedded clusters (a)-(f) depicted in Fig. 5. All values are in eV.

(a)

(b)

(c)

$\mathrm{Li}_{8}{ }^{+} \mathrm{F}_{2}$

$\left|\epsilon_{\text {номо }}\right|$

$E_{\text {ion }}$

$E_{\text {ion }}$

$E_{\text {ion }}$

$E_{\text {ion }}$

$E_{\text {ion }}$
$\Gamma$

E

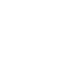

SCF
MCSCF
MR-CISD
MR-CISD + Davidson correction
MR-AQCC
SCF

$\mathrm{F}^{-}$

16.18

12.70

14.20

$\mathrm{Li}_{5}{ }^{+} \mathrm{F}^{-}$

14.93

11.96

13.35

13.19

13.30

0.26
14.90

13.79

13.41

13.31

13.34

0.46

0.56

$\mathrm{Li}_{13}{ }^{+} \mathrm{F}_{4}{ }^{-}$
15.15
14.23
13.42
13.18
13.15
1.1

(d)

(e)

(f)

\begin{tabular}{rrr}
$\mathrm{Li}_{26}{ }^{+} \mathrm{F}_{9}{ }^{-}$ & $\mathrm{Li}_{17}{ }^{+} \mathrm{F}_{5}{ }^{-}$ & $\mathrm{Li}_{25}{ }^{+} \mathrm{F}_{9}{ }^{-}$ \\
14.91 & 14.79 & 14.67 \\
14.50 & 14.02 & 14.19 \\
13.62 & 13.16 & 13.32 \\
13.28 & 12.98 & 12.95 \\
12.99 & 12.89 & 12.63 \\
1.72 & 1.26 & 1.88 \\
\hline
\end{tabular}


TABLE II. Electron affinity of fluorine and orbital energy of the $2 p$ orbital of $\mathrm{F}^{-}$calculated with different basis sets: (a) cc-pVDZ [41], (b) augmented cc-pVDZ (with additional diffuse and polarization functions) $[41,42]$, (c) pseudobasis [44], (d) $(10 s, 7 p) /[2 s, 1 p]$ basis used in Ref. [13].

\begin{tabular}{lrccc}
\hline \hline & (a) & (b) & (c) & (d) \\
\hline$-\epsilon_{2 p} /(\mathrm{eV})$ & 2.25 & 4.93 & 3.59 & -2.72 \\
$E_{a f f}(\mathrm{SCF}) /(\mathrm{eV})$ & -0.16 & 1.40 & 0.76 & -2.72 \\
$E_{a f f}(\mathrm{CI}) /(\mathrm{eV})$ & 1.01 & 3.03 & 1.80 & \\
\hline \hline
\end{tabular}

culation, the molecular orbitals of ground and excited states are optimized simultaneously, the ground-state energy of the state-averaged calculation will, in general, be higher than for a calculation of the ground state only. In the case of the clusters (e) and (f) which contain a fluorine in the center of the surface layer, the difference between the ground-state calculation and the state-averaged calculation for all four (eight) hole configurations is $2.1(2.4) \mathrm{eV}$. The reason for this large energy difference is the fact that for a single-state MCSCF calculation, the hole localizes at the central fluorine and the molecular orbitals at the neighboring fluorines are strongly distorted due to polarization. In the state-averaged calculation, where the orbitals both for the localized and the delocalized hole states are optimized simultaneously, this polarization effect is suppressed. Accordingly, in the case of the clusters (b)-(d), where there is no central fluorine in the surface layer and the ground state is a delocalized hole, the difference between a one-state and a state-averaged MCSCF is small $(<0.1 \mathrm{eV})$. We list in Table I the results for stateaveraged MCSCF for reasons of consistency with calculations for the interaction of an ion with the embedded cluster to be discussed below.

The effect of polarization which reduces the ionization potential should be accounted for by calculations on the CI as well as AQCC levels. These are performed for the neutral cluster employing a single-reference configuration consisting of the molecular orbitals generated by a SCF calculation. For the ionized cluster, we use the molecular orbitals and the reference configurations from the corresponding stateaveraged MCSCF calculation.

The first column of Table I gives the ionization potential $E_{i o n}$ for the simplest "cluster" model possible, a single $\mathrm{F}^{-}$ embedded in a lattice of point charges. Such a model was used in several previous studies of charge transfer $[16,25,28,29]$. The ionization potential of this system can be simply estimated according to first-order perturbation theory as the sum of the electron affinity $E_{a f f}$ of fluorine and the Madelung potential $E_{M}$ at the site of the active fluorine (sum over the Coulomb potentials of the integer point charges). With the literature value $E_{a f f}=3.4 \mathrm{eV}$ and $E_{M}=12.3 \mathrm{eV}$ this would yield $E_{i o n}=15.7 \mathrm{eV}$. The calculated values for $E_{i o n}$ deviate from this value on the different levels of approximation. But a comparison of the first column of Table I with the electron affinities calculated with the same pseudobasis (see Table II in the Appendix) shows that $E_{i o n}$ of the single embedded fluorine is indeed given by $E_{a f f}+E_{M}$ on the different levels of approximation. The large difference between the orbital energy of the HOMO and the MCSCF value (note that for this small system, the MCSCF method coincides with the SCF method since the active space only comprises the $\mathrm{F}_{2 p_{z}}$ orbital) in the first column of Table I reflects the fact that Koopmans' theorem overestimates the electron affinity of fluorine (see the Appendix) while a SCF calculation strongly underestimates it. The CI method is well suited to describe the electron affinity of fluorine (see the Appendix) and yields a value for the ionization potential of the embedded ion which is higher by $1.5 \mathrm{eV}$ than the MCSCF or SCF value. Using a larger basis set, the CI value would be even closer to the above value of $E_{\text {ion }}=15.7 \mathrm{eV}$. This trend highlights the basic dilemma of accurate calculations employing only one active anion in the surface: the simple approximation (SCF) lies fortuitously close to the (experimental) work function for the infinitely extended crystal of $W \approx 12.3 \mathrm{eV}$. However, it is known from the isolated $\mathrm{F}^{-}$ion that only a CI-wave function properly describes the negative ion. But as soon as correlation effects are included on this higher level of approximation, the ionization potential increases by up to $\approx 3 \mathrm{eV}$ and strongly deviates from the experimental value. With a projectile ion in front of the surface, this may give rise to an incorrect ordering of levels and renders any analysis of charge-transfer processes meaningless. Therefore, a larger embedded cluster is required.

The first improvement results from surrounding the active $\mathrm{F}^{-}$with five $\mathrm{Li}^{+}$ions [cluster (a) in Table I]. The inclusion of the nearest-neighbor lithiums in the active cluster allows for more flexibility in the rearrangement of the electron density after ionization, and therefore leads to a reduction of the ionization potential both on the MCSCF and the CI level. Consider the simplest prototype charge-transfer reaction $\left(\mathrm{H}^{+}+\mathrm{LiF}\right)$. Since now the $\mathrm{CI}$ value $E_{\text {ion }}=13.35 \mathrm{eV}$ lies slightly below the recombination energy for $\mathrm{H}^{+}\left(E_{\text {rec }}\right.$ $=13.6 \mathrm{eV}$ ), not only the MCSCF but also the CI calculation would feature the correct level ordering, even though only barely so. For larger basis sets including polarization and diffuse functions, however, the ionization potential will increase and revert the level ordering again.

We turn therefore to clusters with several active fluorines. Different cluster geometries are chosen in order to facilitate the calculation of charge exchange with the projectile ion incident at different sites of the two-dimensional surface unit cell. The first remarkable observation for the larger clusters $[(b)-(f)]$ is the fact that the values for the ionization potential obtained by the CI method (and its size-consistency improvements) are now lower than the MCSCF values. This is because polarization effects within the active cluster are described on the CI level, but not on the MCSCF level where a state-averaged calculation is performed. The polarization effect is visualized in Fig. 6 which shows the difference in the electron density between the CI calculation and the MCSCF calculation for the (lowest) ionized state of the $\mathrm{Li}_{13}{ }^{+} \mathrm{F}_{4}{ }^{-}$ cluster. The density difference is negative at the fluorine sites and positive in the space in between. This means that in the CI calculation, the electron hole is more strongly localized 


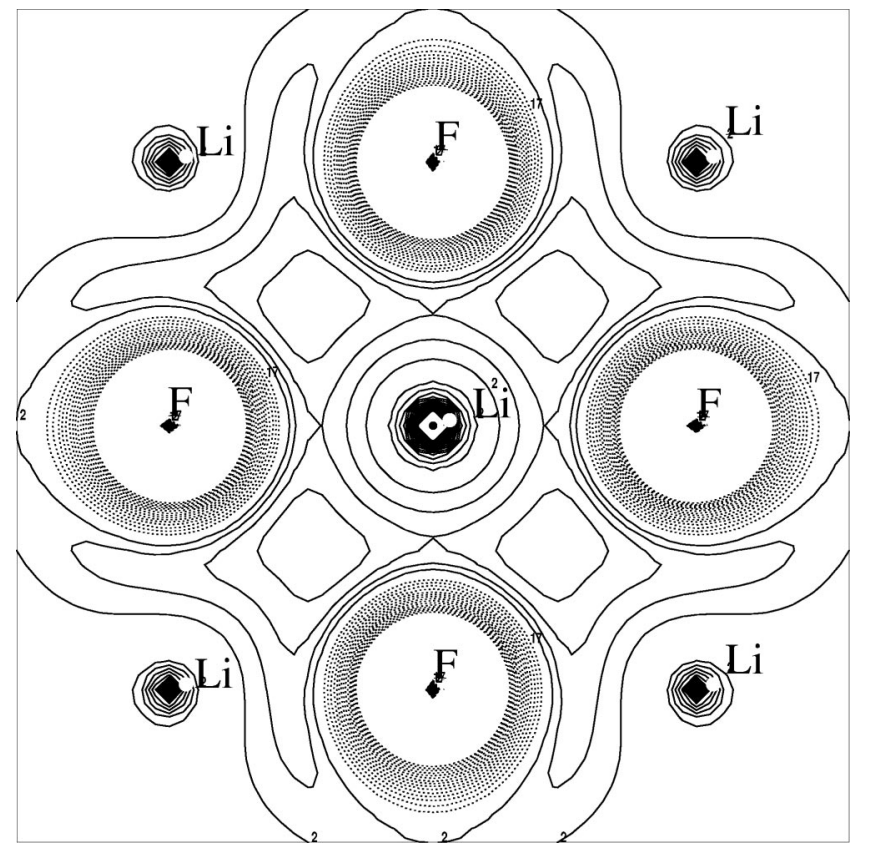

FIG. 6. Contour plot of the electron-density difference $n_{e}^{\mathrm{MR}-\mathrm{CISD}}(\vec{r})-n_{e}^{\mathrm{MCSCF}}(\vec{r})$ between the MR-CISD and the MCSCF approximation for the lowest ionized state. The plot is in the surface plane of the embedded $\mathrm{Li}_{13}{ }^{+} \mathrm{F}_{4}{ }^{-}$cluster [see Fig. 5(c)]. Solid lines, positive density difference; and dotted lines, negative density difference.

around the fluorines with a stronger polarization of the surrounding environment. This polarization effect leads to a lowering of the energy of the ionized state and thereby to a lowering of the ionization potential.

With increasing cluster size, the effects of polarization should increase and lower the value of the ionization potential towards the experimental value of $12.3 \mathrm{eV}$. However, comparing results from cluster pairs (c) and (d) as well as (e) and (f) (Table I) this expectation is not met. The MCSCF value slightly increases due to the fact that we perform an averaging over more states in the larger cluster. The CI calculation violates this expectation because only single and double excitations are computationally feasible to include. This restriction violates size consistency for larger clusters. For the proper description of polarization effects in larger clusters, methods including size-consistency corrections are required. Table I demonstrates that the extended Davidson correction to the CI lowers the ionization potential with respect to the CI values, but can only partially account for the increase of polarization effects with increasing cluster size [the ionization potential rises from $13.18 \mathrm{eV}$ for cluster (c) to $13.28 \mathrm{eV}$ for cluster (d) but decreases from 12.98 for cluster (e) to $12.95 \mathrm{eV}$ for cluster (d)]. The MR-AQCC method properly describes the increase of polarization effects with increasing cluster size: the ionization potential decreases from $13.15 \mathrm{eV}$ for cluster (c) to $12.99 \mathrm{eV}$ for cluster (d) and from $12.89 \mathrm{eV}$ for cluster (e) to $12.63 \mathrm{eV}$ for cluster (f), thereby approaching the experimental value of the work function of $12.3 \mathrm{eV}$. Only in the limit of very large clusters, the correct value of the work function can be reached, since the polarization of the crystal after removal of one electron from the surface is a long-range effect.

The ionization potentials of the larger clusters demonstrate that $\mathrm{CI}$ and its size-consistent improvements are better suited to yield the correct work function of $\mathrm{LiF}$ than a simple MCSCF calculation. However, complete convergence with respect to the choice of basis set and with respect to cluster size is computationally very demanding to achieve due to the long-range nature of the polarization effects. A possible way to overcome this problem and keeping the active cluster small would be to include polarization of a larger part of the crystal by an improved embedding scheme of the cluster [11]. Another route could be along a recent work of Govind et al. [45] where a small cluster is described on the CI level and surrounded by a larger cluster described by densityfunctional theory. However, both approaches have not yet been implemented in the framework of a MR-CI calculation that yields orthonormalized wave functions for ground and excited states.

The present choice for cluster size and basis, which will be used in the following section for calculating the interaction with a projectile ion, is clearly a compromise between two complementary requirements which cannot be fulfilled simultaneously with available $a b$ initio methods: (i) the correct value of the work function which is connected to the correct asymptotic level ordering of the combined ionsurface system and (ii) the correct multireference electronic wave function of the surface cluster. The latter allows one to compute the coupling-matrix elements between ion and surface in a complete time-dependent solution of the problem and thereby may strongly influence the efficiency of the charge-transfer process. It is also worth noting that potential surfaces for charge exchange pose a far greater challenge than most previous applications of the embedded-cluster approach [7-11], in that an electron "leaves" the crystal by being transferred to the projectile instead of "just" being excited to a state which is still localized inside the crystal. This induces a much stronger polarization of the environment and complicates a correct description of the crystalline environment.

It is of interest to compare the present results for $E_{i o n}$ with the input to previous ion-surface calculations where orbital energies have been used to discuss charge transfer $[12,13,15]$. Table I lists the orbital energies of the HOMO, $\left|\epsilon_{\text {Номо }}\right|$, of the neutral closed-shell system. The identification of this energy with the ionization potential according to Koopmans' theorem is poorly justified for systems that involve negative ions. Accordingly, $\left|\epsilon_{\text {НOMO }}\right|$ is up to $3.5 \mathrm{eV}$ larger than the ionization potentials according to the MCSCF method, corresponding to an artificial downwards shift of the valence-band edge. In the orbital energy picture, the level ordering is reversed. This effect can be observed in the calculation of Ref. [12] for $\mathrm{H}^{+}+\mathrm{LiCl}$, where the orbital energy of the $\mathrm{Cl}_{3 p}$ valence states of $\mathrm{LiCl}$ is below the hydrogenic level. At first glance surprisingly, in Ref. [13] a value of $\epsilon_{\text {Номо }}=-10.61 \mathrm{eV}$ was obtained for the orbital energies of the HOMO for both the $\mathrm{Li}_{5}{ }^{+} \mathrm{F}^{-}$and the $\mathrm{Li}^{+} \mathrm{F}_{5}{ }^{-}$embedded cluster representing a $\mathrm{LiF}$ surface. This high value for the valence-band edge can, however, be traced back to 


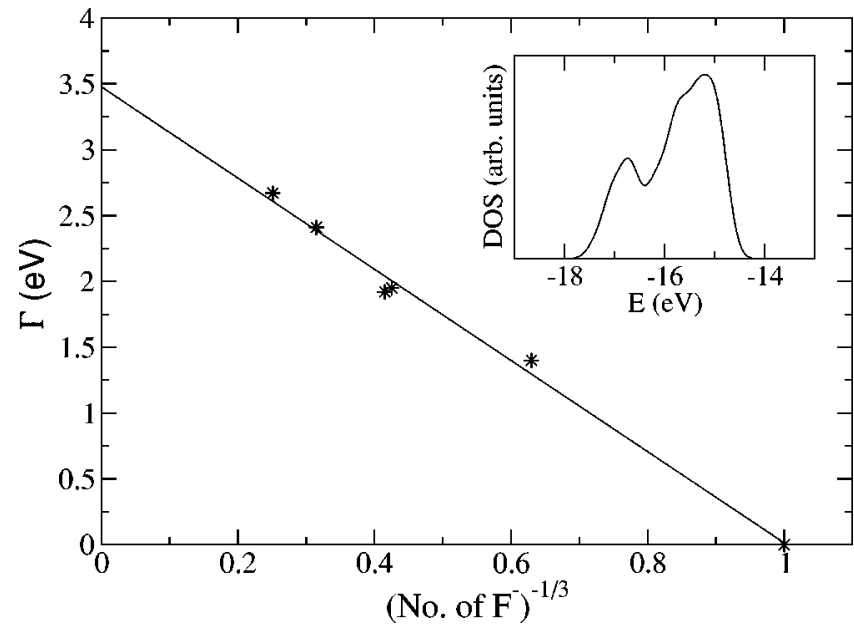

FIG. 7. Bandwidth (difference between the orbital energies of the highest and lowest $\mathrm{F}_{2 p}$-like orbitals) of bulk embedded $\mathrm{LiF}$ clusters as a function of the inverse linear dimension $\left[\propto\left(\text { number of } \mathrm{F}^{-}\right)^{-1 / 3}\right]$ of the cluster. Inset: average of the density of $\mathrm{F}_{2 p}$ states in the $\mathrm{Li}_{135}{ }^{+} \mathrm{F}_{62}{ }^{-}$and $\mathrm{Li}_{146}{ }^{+} \mathrm{F}_{63}{ }^{-}$clusters. Each discrete state is represented by a Gaussian peak with a full width at half maximum of $0.4 \mathrm{eV}$.

the very small basis for $\mathrm{F}$ which yields a negative electron affinity (Table II) and the use of an odd number of total charges for the embedding.

The last row of Table I gives the bandwidth $\Gamma$ of the embedded clusters, calculated as the orbital energy difference in the SCF calculation of the neutral closed-shell system between the highest and lowest $\mathrm{F}_{2 p}$-like orbital. $\Gamma$ is another important figure of merit of electronic structure calculations for surfaces even though, for the charge-transfer process, and in particular for its energetic threshold behavior, the width is less important than the ionization energy for electrons from the upper band edge. If the active cluster contains only one fluorine, the "bandwidth" is the energy difference between the $2 p_{x}$ or $2 p_{y}$ orbitals that are in the surface plane and the $2 p_{z}$ orbital that sticks out of the surface. For clusters with several fluorines, the linear combination of the atomic orbitals leads to an additional splitting which reaches already more than half the experimental bandwidth (of $3.4 \mathrm{eV}$ ) for the largest clusters evaluated in Table I. Convergence of $\Gamma$ as a function of size is slow.

In order to check if the embedded-cluster approach can reproduce the bandwidth of the LiF crystal in the limit of an infinitely extended crystal, we have performed Hartree-Fock calculations of bulk embedded clusters of increasing size up to $\mathrm{Li}_{146}{ }^{+} \mathrm{F}_{63}{ }^{-}$[46]. Figure 7 shows the bandwidth $\Gamma$ as a function of the number of fluorine ions in the cluster. Under the assumption that the width is determined by the linear dimension of the cluster, i.e., it is a function of (number of $\left.\mathrm{F}^{-}\right)^{1 / 3}$, we extrapolated the bandwidth to infinite cluster size by plotting $\Gamma$ as a function of (number of $\left.\mathrm{F}^{-}\right)^{-1 / 3}$. The resulting linear curve crosses the ordinate at $3.5 \mathrm{eV}$ which is close to the experimental bandwidth of $3.4 \mathrm{eV}$. The inset of Fig. 7 demonstrates that employing Gaussian broadening of the discrete levels, the density of states of finite embedded clusters converges towards
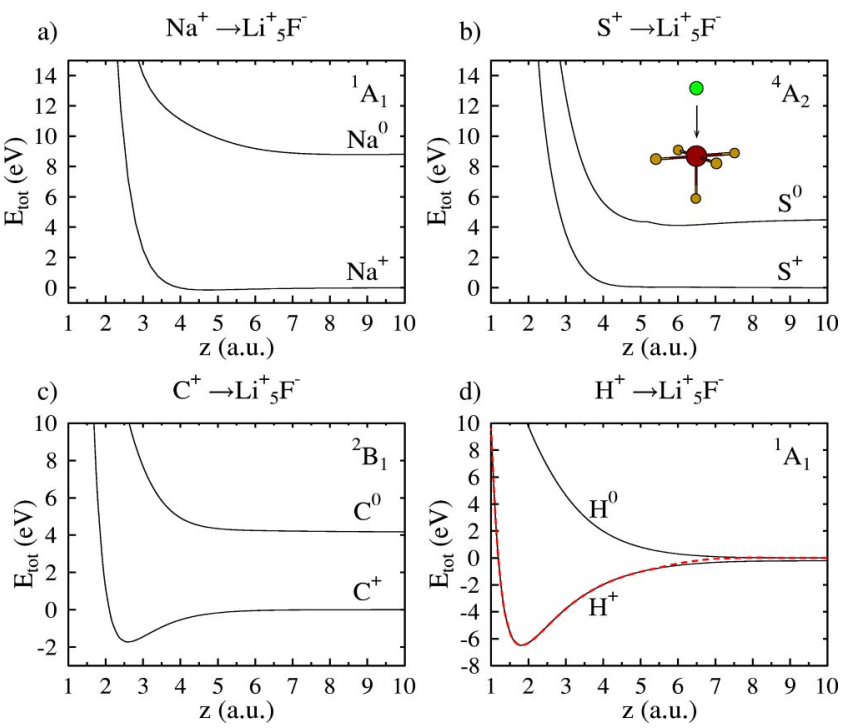

FIG. 8. Cut through potential energy surfaces on the MR-CI level for different ions. The ion approaches an embedded $\mathrm{Li}_{5}{ }^{+} \mathrm{F}^{-}$ cluster in vertical incidence with touch down on the fluorine. The dashed line indicates the diabatic curve of the ionic configuration for an avoided crossing, if existent. The "kink" in the covalent curve for sulfur $[\operatorname{panel}(\mathrm{b})]$ is due to the interaction between the lowest covalent and higher-lying ionic states.

the density of states of the (bulk) LiF valence band (compare the experimental data of Ref. [27]).

\section{INTERACTION OF SINGLY CHARGED PROJECTILE IONS WITH EMBEDDED SURFACE CLUSTERS}

We consider now the collision system with the projectile approaching the cluster. As we are interested in adiabatic potential surfaces, we consider the position vector $\vec{R}$ of the ion relative to the $\mathrm{LiF}$ surface to be a fixed parameter. The ion can touch down (in normal incidence) anywhere in the surface unit cell. We choose three representative positions corresponding to three different cuts through the energy surface: on top of a fluorine, on top of a lithium, and at the midpoint in between neighboring $\mathrm{F}^{-}$ions (see Fig. 4). These three geometries possess the $\mathrm{C}_{2 v}$ point-group symmetry which facilitates the calculation. As projectile ions we consider ions with different recombination energies which were used in the experiment of Ref. [23]: $\mathrm{Na}^{+}$(E recomb $=5.14 \mathrm{eV}), \mathrm{S}^{+}(10.36 \mathrm{eV}), \mathrm{C}^{+}(11.26 \mathrm{eV})$, and $\mathrm{H}^{+}(13.6$ $\mathrm{eV})$.

Figure 8 presents energy curves for the interaction of the different ion species with the embedded $\mathrm{Li}_{5}{ }^{+} \mathrm{F}^{-}$surface cluster at the MR-CISD level [48]. In each case two configurations are displayed: the "ionic" configuration, representing the entrance channel and the covalent state converging towards a neutralized projectile representing the exit channel. The latter represents a covalent bond with the surface fluorine at small distances [49]. The configuration of the embedded cluster without the projectile is a spin singlet with $A_{1}$ symmetry $\left({ }^{1} A_{1}\right)$. Therefore, the configuration of the com- 
bined cluster-projectile system is determined by the groundstate configuration of the projectile ion. For $\mathrm{Na}^{+}$and $\mathrm{H}^{+}$ with ${ }^{1} \mathrm{~S}$ configuration this yields ${ }^{1} A_{1}$ for the combined system. The ground state of a $\mathrm{S}^{+}$ion is ${ }^{4} \mathrm{~S}$ and the combined system is a quadruplet with $A_{2}$ symmetry $\left({ }^{4} A_{2}\right)$. In the ${ }^{2} P$ configuration of the $\mathrm{C}^{+}$ion, a single electron occupies one of the $2 p$ orbitals. This gives rise to two different possibilities for the combined system: ${ }^{2} A_{1}$ and ${ }^{2} B_{1}$ (the ${ }^{2} B_{2}$ configuration is equivalent to ${ }^{2} B_{1}$ ). Since the ground state of the $\mathrm{CF}$ molecule is ${ }^{2} \Pi[50]$, the ionic state is more likely to interact with the covalent state in the ${ }^{2} B_{1}$ configuration which we have consequently chosen for our calculations involving carbon projectiles.

The energy of the entrance channel, i.e., the ionic state at large distance $(R \rightarrow \infty)$, is chosen as zero for the total energy. The energy of the lowest covalent state at large distance can be approximately determined as the difference between the ionization potential of the cluster (see Table I) and the recombination energy of the projectile ion. For $\mathrm{Na}^{+}$approaching $\mathrm{Li}_{5}{ }^{+} \mathrm{F}^{-}$[Fig. 8(a)], this leads to a difference between ionic and covalent states of about $8.5 \mathrm{eV}$ at large distance. At smaller distances, the difference even increases and no apparent interaction between the two potential surfaces is visible. Neutralization of $\mathrm{Na}^{+}$is therefore very unlikely to occur in slow collisions with LiF. A small probability for charge exchange at higher collision energies via a kinematic resonance [51,52], can, however, not be excluded. In fact, Meyer et al. have observed a small neutral fraction of $\approx 5 \%$ in grazing scattering of $5-\mathrm{keV} \mathrm{Na}^{+}$ions at a $\mathrm{LiF}(100)$ surface [53].

The covalent energy surface for $\mathrm{S}^{+} \rightarrow \mathrm{Li}_{5}{ }^{+} \mathrm{F}^{-}$[Fig. 8(b)] lies asymptotically $4.5 \mathrm{eV}$ above the entrance channel and displays only a very small inclination towards the ionic state at smaller distances. For trajectories following this particular section through the potential surface, the existence of an efficient charge-transfer channel can be ruled out. We will see in the following, however, that along other cuts, and at small distances, a charge-transfer channel opens up. Similar observations apply to $\mathrm{C}^{+} \rightarrow \mathrm{Li}_{5}{ }^{+} \mathrm{F}^{-}$[Fig. 8(c)].

A qualitatively different situation arises for $\mathrm{H}^{+}$ $\rightarrow \mathrm{Li}_{5}{ }^{+} \mathrm{F}^{-}$[Fig. 8(d)]. The ionization potential of the cluster $(13.35 \mathrm{eV}$, see Table I) is slightly smaller than the recombination energy of $\mathrm{H}^{+}$. The covalent configuration lies below the ionic configuration which results in an avoided crossing at about $R=6.5$ a.u. We indicate the crossing by a dashed line for the diabatic curve [54]. We note, however, that the position of the avoided crossing is strongly dependent on the basis and on the level of approximation. In fact, employing a larger basis set for the $\mathrm{F}^{-}$ion leads to a wrong ordering of the ionic and covalent states at large distances and eliminates the avoided crossing.

Considering now the cluster $\mathrm{Li}_{8}{ }^{+} \mathrm{F}_{2}{ }^{-}$(Fig. 9) with two covalent ${ }^{1} A_{1}$ configurations representing a different cut through the energy surface with impact at the midpoint between two surface atoms, the ionic level of $\mathrm{H}^{+}$, is located in between the covalent states at large distances [Fig. 9(d)]. An avoided crossing between ionic and covalent states is visible
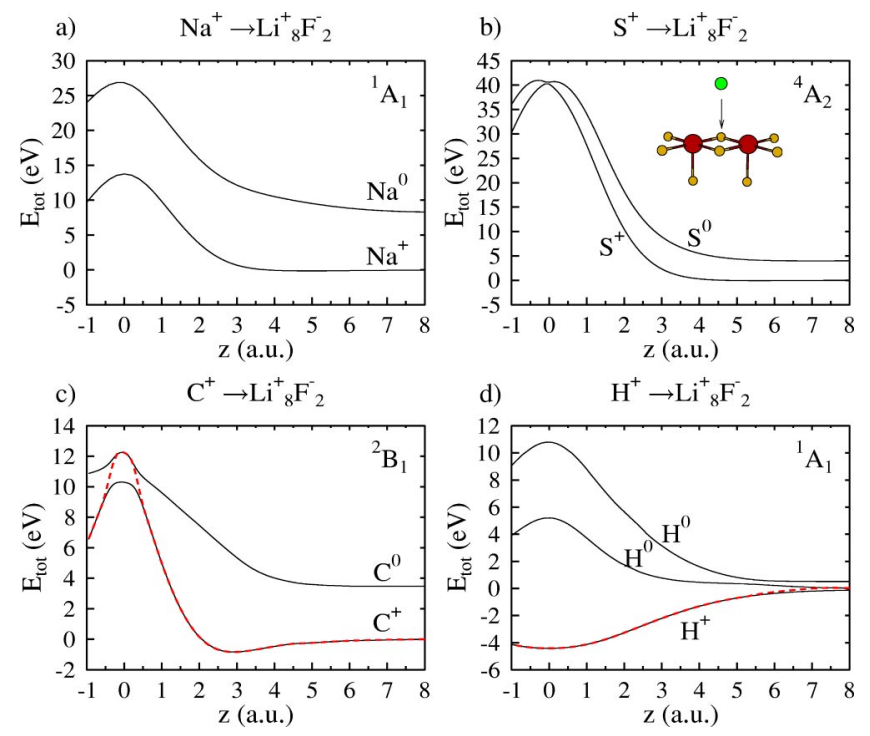

FIG. 9. Cuts through potential-energy surfaces (MR-CI level) for different ions approaching an embedded $\mathrm{Li}_{8}{ }^{+} \mathrm{F}_{2}{ }^{-}$cluster (vertical incidence, touch down at midpoint between $\mathrm{Li}$ and $\mathrm{F}$ ).

where the diabatic curve connects the lowest state in energy with the next higher state.

For $\mathrm{C}^{+} \rightarrow \mathrm{Li}_{8}{ }^{+} \mathrm{F}_{2}{ }^{-}$[Fig. 9(c)], there is a clear sign of an avoided crossing between the ionic and the lowest covalent state shortly before and after the projectile crosses the surface plane. We have continued to calculate the curves to negative $z$ (i.e., below the surface) since for this geometry the ion can penetrate into the surface. We have also performed calculations where the ion is incident not exactly on the center of the cluster but closer to either an $\mathrm{F}^{-}$or $\mathrm{Li}^{+}$ion. In both cases, the avoided crossings become narrower and the positions are shifted away from the surface plane.

For $\mathrm{S}^{+} \rightarrow \mathrm{Li}_{8}{ }^{+} \mathrm{F}_{2}{ }^{-}$[Fig. 9(b)], the two avoided crossings (above and below the surface) reduce to a half-sided avoided crossing between the lower covalent and the ionic curve in the surface plane. This clearly facilitates charge exchange while the sulfur ion penetrates into the surface. Note, however, the large energy scale in this panel. A kinetic energy of about $40 \mathrm{eV}$ is necessary to overcome the repulsion between the electron cloud of $\mathrm{S}^{+}$and the electron clouds of the surface ions if the lattice is kept frozen. If one takes the molecular dynamics of the lattice into account, the projectile ion pushes the surface ions along or to the side and can penetrate with lower kinetic energy. Finally, for $\mathrm{Na}^{+}$approaching the surface an avoided crossing is absent even if surface penetration is taken into account. We therefore can conclude that charge transfer to $\mathrm{Na}^{+}$is suppressed in near adiabatic hyperthermal scattering with a LiF surface.

For completeness, we show in Fig. 10 potential curves corresponding to a touch-down point on top of a surface $\mathrm{Li}^{+}$ ion. In the case of $\mathrm{S}^{+} \rightarrow \mathrm{Li}_{13}{ }^{+} \mathrm{F}_{4}{ }^{-}$, there are four covalent levels of $A_{2}$ symmetry. Since the ionic energy curve is parallel to the covalent ones and since no configurational mixing occurs in the CI wave functions, charge exchange is not likely to take place in this geometry. Therefore, we can conclude for singly charged sulfur ions that charge exchange is most likely to occur when the ion is vertically incident on 

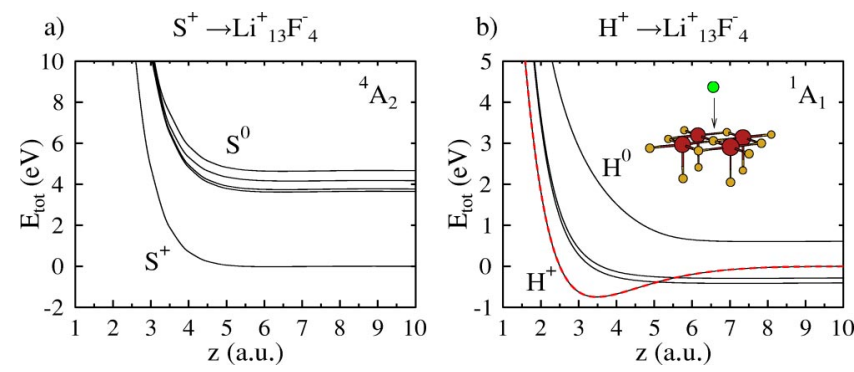

FIG. 10. Cuts through potential-energy surfaces (MR-CI level) for different ions approaching an embedded $\mathrm{Li}_{13}{ }^{+} \mathrm{F}_{4}{ }^{-}$cluster (vertical incidence, touch down on $\mathrm{Li}$ ).

interstitial sites in the surface and not when it is incident on either lithium or fluorine ions. For $\mathrm{H}^{+} \rightarrow \mathrm{Li}_{13}{ }^{+} \mathrm{F}_{4}{ }^{-}$, in contrast, the ionic state is embedded in the center of the covalent band at large distances and displays two very narrow avoided crossings. Charge exchange can take place for hydrogen ions incident on any site of the surface.

The potential-energy curves presented above confirm (within the limitations of the embedded-cluster approach discussed in the preceding section) the model of defectmediated sputtering for the ablation of secondary particles from a LiF surface under the impact of slow singly charged ions $[23,25]$. For the ion species where sputtering has been experimentally observed, i.e., for $\mathrm{S}^{+}, \mathrm{C}^{+}$, and $\mathrm{H}^{+}$, Figs. 8-10 delineate pathways for charge transfer as a precursor event for the creation of a self-trapped hole. For $\mathrm{H}^{+}$, clear evidence for Landau-Zener avoided crossings are visible in the potential-energy curves at any touch-down point, provided that the cluster is large enough to lead to an embedding of the ionic state into the set of covalent levels at large distance. For $\mathrm{S}^{+}$and $\mathrm{C}^{+}$, pathways to charge transfer are open in at least a restricted domain of impact geometries. For $\mathrm{Na}^{+}$, by contrast, no avoided crossing materializes in any geometry since the projectile level stays above the valence band at all distances. Consequently, no potential sputtering is expected for this ion species, which is in agreement with the experiment [23]. Our calculation thus confirms the threshold behavior as a function of recombination energy for charge transfer and thus for hole formation as precursor for potential sputtering. Clearly, in order to assess the efficiency for charge transfer at the different sites, a full dynamical calculation using the nonadiabatic coupling elements will be required, which is outside the scope of the present paper.

\section{INTERACTION OF $\mathrm{H}^{+}$WITH A LARGE SURFACE CLUSTER}

The interplay between cluster size, the effect of polarization and correlation, and level of feasible sophistication of embedded cluster calculations has already been alluded to in Sec. III. For larger clusters, the bandwidth converges towards the experimental value of $\Gamma=3.4 \mathrm{eV}$, but the valence-band edge $\left(E_{i o n}\right)$ remains $1-2 \mathrm{eV}$ too low. As a prototype example we present now results for the largest cluster we have been able to treat on the MR-CI level interacting with the simplest
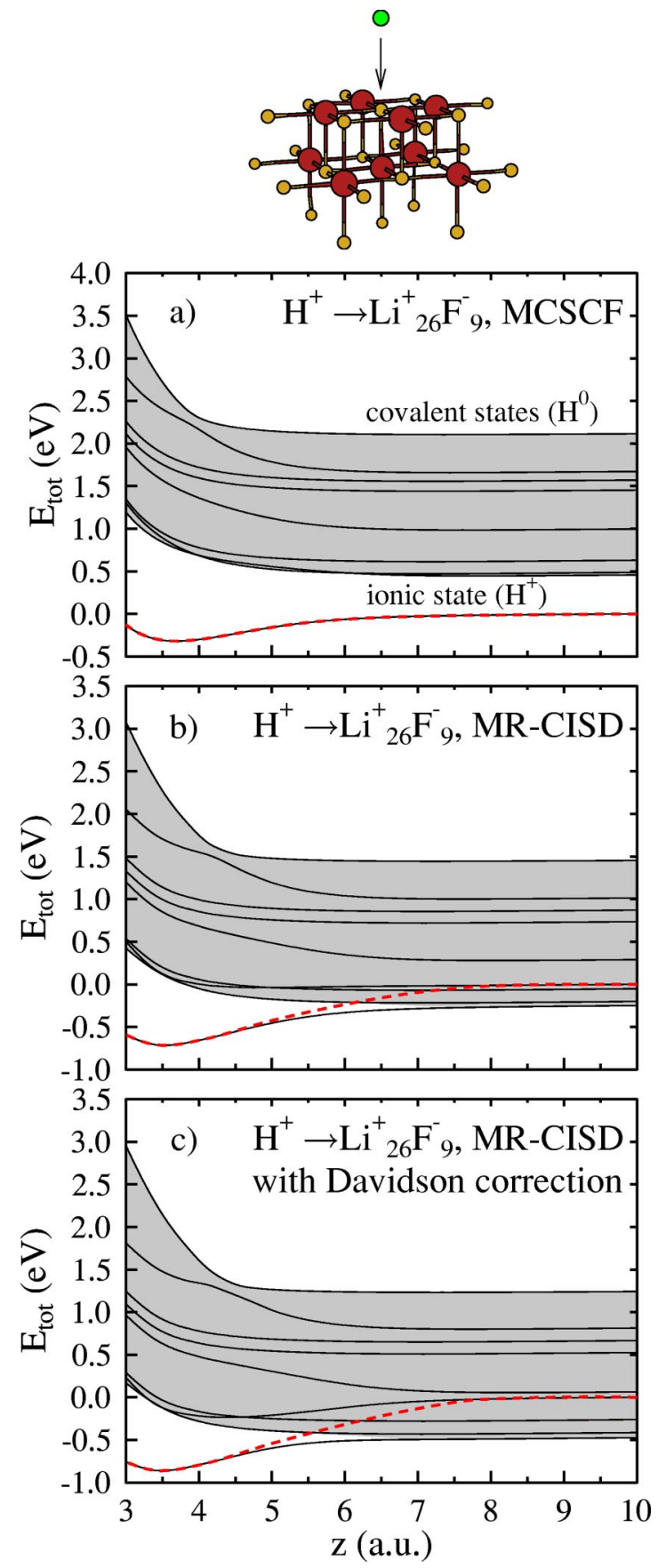

FIG. 11. Cuts through potential-energy surfaces for $\mathrm{H}^{+}$approaching an embedded $\mathrm{Li}_{26}{ }^{+} \mathrm{F}_{9}{ }^{-}$cluster (vertical incidence, touch down on Li). Comparison of different levels of approximation: (a) MCSCF, (b) MR-CI, and (c) MR-CI with Davidson correction.

projectile, $\mathrm{H}^{+}$. Figure 11 presents cuts through the potentialenergy surfaces for a proton touching down on $\mathrm{Li}$ on three different levels of approximation: MCSCF, MR-CI, and MR-CI with Davidson correction $[19,20]$. The $F_{2 p}$ atomic orbitals split into eight valence orbitals of $A_{1}$ symmetry from which an electron can be transferred to the $\mathrm{H}^{+}$ion. These eight states represent the valence band of $\mathrm{LiF}$ shaded in gray.

On the MCSCF level, the covalent states are all higher in energy than the ionic state which does not interact with any 
of the covalent states. On the MR-CI level (with single and double excitations), correlation and polarization effects are included and the covalent levels are shifted down by $0.75 \mathrm{eV}$ with respect to the ionic level. (This is similar to the energy difference of $0.88 \mathrm{eV}$ between the MCSCF and the CI calculations of the ionization potential of the embedded $\mathrm{Li}_{26}{ }^{+} \mathrm{F}_{9}{ }^{-}$cluster in Table I). The shift due to the correlation energy leads to an avoided crossing between the ionic entrance channel and some of the covalent states representing the exit channel. The dashed line indicates the diabatic energy curve of the ionic state which crosses several of the covalent curves. Since in large clusters, the correlation energy is often underestimated, we also apply the Davidson correction $[19,20]$ to approximately correct for size consistency. The Davidson correction affects the covalent states more than the ionic state and leads to an additional downward shift of the covalent states by $0.25 \mathrm{eV}$. The ionic state is now clearly embedded into the band of covalent states. The energetic difference between the asymptotic ionic and lowest covalent level is found to be $0.5 \mathrm{eV}$ compared to the experimental value of $1.3 \mathrm{eV}$, while the width of $\Gamma=1.75 \mathrm{eV}$ (without extrapolation of cluster size) is still about $1.5 \mathrm{eV}$ smaller than the experimental value. We also performed a calculation of the level ordering at large projectile-surface distance on the AQCC level. The resulting energy difference between the lowest covalent and the ionic level is $0.63 \mathrm{eV}$. This value is a little larger than the value of $0.5 \mathrm{eV}$ of the MR-CI with Davidson correction, and confirms the expectation that methods containing size-consistency corrections such as AQCC should yield converged potential-energy curves for charge exchange, provided that a calculation with larger cluster size and basis set becomes numerically feasible with further advances in computing power.

\section{CONCLUSION AND OUTLOOK}

We have presented energy surfaces relevant for charge transfer between singly charged ions and a LiF surface. Different avenues of $a b$ initio calculations are explored and limitations that apply to currently feasible methods and system sizes are delineated. One key observation is the failure of the frequently used SCF method to predict the absolute position of the upper valence-band edge, i.e., the work function of the system. This leads asymptotically to a wrong level ordering for the projectile-surface systems and raises doubts as to the use of SCF wave functions as input for a dynamical charge-transfer calculation. More reliable potential-energy curves can be obtained by a multireference configurationinteraction calculation. This is a step towards the inclusion of polarization and correlation effects upon taking an electron out of the surface. However, convergence of this method is limited by the restriction to a finite number of excitations that can be included in the expansion and by size inconsistency of the method for larger systems. The Davidson correction to the CI and - even more-methods containing sizeconsistency effects such as AQCC yield a considerable improvement in the inclusion of correlation and polarization effects.
Another limitation results from the representation of an ionic crystal surface by an embedded cluster which reduces an infinite system to a finite-size system without periodicity. The continuous band reduces to a finite number of discrete states and the bandwidth is typically underestimated. As charge-transfer processes are strongly localized and are primarily sensitive to the upper band edge, this limitation is less of a concern.

Clearly, fully converged calculations including both correlation and polarization effects and the features of an infinitely extended system remain a challenge. Keeping these restrictions in mind, the present calculation can provide an ab initio confirmation of the recently observed threshold behavior for potential sputtering via formation of self-trapped holes. Our calculations demonstrate the existence of chargetransfer channels for the neutralization of slow $\mathrm{S}^{+}, \mathrm{C}^{+}$, and $\mathrm{H}^{+}$ions incident on a $\mathrm{LiF}$ surface, while for $\mathrm{Na}^{+}$ions this channel is closed. This explains the recently measured threshold of $\simeq 10 \mathrm{eV}$ for the potential sputtering of LiF corresponding to the recombination energy of $\mathrm{S}^{+}$ions.

\section{ACKNOWLEDGMENTS}

The work was supported by the Austrian Science Fund Project Nos. P12470-TPH (L.W. and J.B.) and P14442-CHE (M.D., Th.M., and H.L.) and by the Special Research Program F16. L.W. and J.B. also acknowledge support by EU Project No. HPRI-2001-50036. We acknowledge stimulating discussions with A. Shluger, P. Sushko, S. Pantelides, C. O. Reinhold, P. S. Krstic, A. G. Borisov, and N. March.

\section{APPENDIX: BASIS SETS AND ELECTRON AFFINITY OF F}

The choice of the basis set for fluorine is essential for a proper description of the electron affinity. Table II presents results for different basis sets. The affinity is calculated as the difference in total energy of the negative ion and the neutral fluorine. We compare a SCF calculation with a single-reference CI calculation with single and double excitations. Clearly, only a CI calculation with a basis set including diffuse and polarization functions gives a result close to the literature value of $3.4 \mathrm{eV}$ [55]. The SCF level where the six valence electrons occupy three equivalent $2 p$ orbitals is not well suited to describe negative ions. For basis sets without diffuse and polarization functions [such as (a) and d)] the affinity even becomes negative. For our calculations involving clusters with several fluorines, we use the pseudobasis (c). In this basis, the $1 s$ orbital is described by a pseudopotential. Only two contracted basissets are used for the description of each the $2 s$ and $2 p$ orbitals.

Nevertheless, the basis yields a positive value for the affinity on the SCF level, and on the CI level it accounts for more than half of the electron affinity. This is a compromise between using a cluster that contains only one fluorine which is described very accurately with a large basis set as in Ref. [16] and using a larger cluster with a small basis as in Ref. [13]. The minimal basis of Ref. [13] contains only one basis function for each occupied orbital and no basis functions for 
virtual orbitals that would allow a CI calculation. The resulting electron affinity is strongly negative and the SCF wave functions certainly not suitable for charge-exchange calculations. Table II also demonstrates that the orbital energy of the $2 p$ orbitals of $\mathrm{F}^{-}$is not a reliable mea-sure for $E_{\text {aff }}$ : $\left|\epsilon_{\text {Номо }}\right|$ yields values up to $1.6 \mathrm{eV}$ higher than the experi- mental value of the electron affinity and $3.2 \mathrm{eV}$ higher than the SCF value of the electron affinity. The underlying reason for this failure of Koopmans' theorem is that in the SCF calculation all six $2 p$ electrons are forced into equivalent orbitals, whereas a CI wave function shows a strong admixture of configurations with excitations into higher orbitals.
[1] See, e.g., A. Arnau, F. Aumayr, P.M. Echenique, M. Grether, W. Heiland, J. Limburg, R. Morgenstern, P. Roncin, S. Schippers, R. Schuch, N. Stolterfoht, P. Varga, T.J.M. Zouros, and HP. Winter, Surf. Sci. Rep. 27, 113 (1997), and references therein.

[2] B.H. Bransden and M.R.C. McDowell, Charge Exchange and the Theory of Ion-Atom Collisions (Clarendon Press, Oxford, 1992).

[3] J.B. Delos, Rev. Mod. Phys. 53, 287 (1981).

[4] M. Kimura and N.F. Lane, Adv. At., Mol., Opt. Phys. 26, 79 (1990).

[5] S.A. Deutscher, X. Yang, and J. Burgdörfer, Phys. Rev. A 55, 466 (1997); P. Kürpick, U. Thumm, and U. Wille, ibid. 56, 543 (1997); P. Nordlander and J.C. Tully, Phys. Rev. B 42, 5564 (1990).

[6] C. Lemell, X.-M. Tong, F. Krausz, and J. Burgdörfer, Phys. Rev. Lett. 90, 076403 (2003).

[7] A.Y.S. Kung, A.B. Kunz, and J.M. Vail, Phys. Rev. B 26, 3352 (1982).

[8] N.W. Winter, R.M. Pitzer, and D.K. Temple, J. Chem. Phys. 86, 3549 (1987).

[9] H. Tatewaki and E. Miyoshi, Surf. Sci. 327, 129 (1995); H. Tatewaki, Phys. Rev. B 60, 3777 (1999).

[10] M.A. Nygren, L.G.M. Pettersson, Z. Barandiarán, and L. Seijo, J. Chem. Phys. 100, 2010 (1994).

[11] P.V. Sushko, A.L. Shluger, and C.R.A. Catlow, Surf. Sci. 450, 153 (2000).

[12] R. Souda, K. Yamamoto, W. Hayami, B. Tilley, T. Aizawa, and Y. Ishizawa, Surf. Sci. 324, L349 (1995).

[13] E.A. García, P.G. Bolcatto, M.C.G. Passeggi, and E.C. Goldberg, Phys. Rev. B 59, 13370 (1999).

[14] R. Brako and D. Newns, Surf. Sci. 108, 253 (1980).

[15] P.A. Zeijlmans van Emmichoven, A. Niehaus, P. Stracke, F. Wiegershaus, S. Krischok, V. Kempter, A. Arnau, F.J. García de Abajo, and M. Peñalba, Phys. Rev. B 59, 10950 (1999).

[16] A.G. Borisov, V. Sidis, and H. Winter, Phys. Rev. Lett. 77, 1893 (1996); A.G. Borisov and V. Sidis, Phys. Rev. B 56, 10 628 (1997); S.A. Deutscher, A.G. Borisov, and V. Sidis, Phys. Rev. A 59, 4446 (1999).

[17] R. Shepard, The Multiconfiguration Self-consistent Field Method, Advances in Chemical Physics, Ab Initio Methods in Quantum Chemistry II, Vol. LXIX (Wiley, Hoboken, NJ, 1987), pp. 63-200.

[18] I. Shavitt, Methods of Electronic Structure Theory, 3rd ed., edited by H.F. Schaefer (Plenum, New York, 1977), p. 189.

[19] S.R. Langhoff and E.R. Davidson, Int. J. Quantum Chem. 8, 61 (1974)

[20] P.J. Bruna, S.D. Peyerimhoff, and R.J. Buenker, Chem. Phys. Lett. 72, 278 (1981)
[21] P.G. Szalay and R.J. Bartlett, Chem. Phys. Lett. 214, 481 (1993); J. Chem. Phys. 103, 3600 (1998).

[22] R.J. Bartlett and G.D. Purvis III, Phys. Scr. 21, 255 (1980).

[23] G. Hayderer, M. Schmid, P. Varga, HP. Winter, F. Aumayr, L. Wirtz, C. Lemell, J. Burgdörfer, L. Hägg, and C.O. Reinhold, Phys. Rev. Lett. 83, 3948 (1999).

[24] F. Aumayr, J. Burgdörfer, P. Varga, and HP. Winter, Comments At. Mol. Phys. 34, 201 (1999).

[25] L. Wirtz, G. Hayderer, C. Lemell, J. Burgdörfer, L. Hägg, C.O. Reinhold, P. Varga, HP. Winter, and F. Aumayr, Surf. Sci. 451, 197 (2000).

[26] Y. Wang, P. Nordlander, and N.H. Tolk, J. Chem. Phys. 89, 4163 (1988).

[27] F.J. Himpsel, L.J. Terminello, D.A. Lapiano-Smith, E.A. Eklund, and J.J. Barton, Phys. Rev. Lett. 68, 3611 (1992).

[28] L. Hägg, C.O. Reinhold, and J. Burgdörfer, Phys. Rev. A 55, 2097 (1997).

[29] L. Wirtz, C.O. Reinhold, C. Lemell, and J. Burgdörfer, Phys. Rev. A 67, 012903 (2003).

[30] A.B. Kunz, Phys. Rev. B 26, 2056 (1982).

[31] P. Fulde, Electron Correlations in Molecules and Solids (Springer, New York, 1995).

[32] J. Burgdörfer, in Review of Fundamental Processes and Applications of Atoms and Ions, edited by C.D. Lin (World Scientific, Singapore, 1993), p. 517.

[33] Since the coupling-matrix elements are frequently strongly peaked as a function of the internuclear distance, their direct use in the expansion of the time-dependent Schrödinger equation (2.2) may not be numerically feasible. One customarily performs a unitary transformation from the adiabatic basis to a "diabatic basis" which is defined such that the off-diagonal coupling-matrix elements are zero. In this case, the offdiagonal matrix elements of the Hamiltonian $\hat{H}$ are nonzero and cause the coupling between different diabatic states.

[34] L.D. Landau, Phys. Z. Sowjetunion 1, 46 (1932); C. Zener, Proc. R. Soc. London, Ser. A 137, 696 (1932); E.C.G. Stückelberg, Helv. Phys. Acta 5, 369 (1932).

[35] N. Rosen and C. Zener, Phys. Rev. 40, 502 (1932); N.Y. Demkov, Dokl. Akad. Nauk SSSR 166, 1076 (1966).

[36] G. Hirsch, P.J. Bruna, R.J. Buenker, and S.D. Peyerimhoff, Chem. Phys. 45, 335 (1980).

[37] H. Lischka, R. Shepard, I. Shavitt, F.B. Brown, R.M. Pitzer, R. Ahlrichs, H.-J. Böhm, A.H.H. Chang, D.C. Comeau, R. Gdanitz, H. Dachsel, M. Dallos, C. Erhard, M. Ernzerhof, G. Gawboy, P. Höchtl, S. Irle, G. Kedziora, T. Kovar, Th. Müller, V. Parasuk, M. Pepper, P. Scharf, H. Schiffer, M. Schindler, M. Schüler, E. Stahlberg, P.G. Szalay and J.-G. Zhao, computer code COLUMBUS, Release 5.7 (2000).

[38] R. Shepard, I. Shavitt, R.M. Pitzer, D.C. Comeau, M. Pepper, H. Lischka, P.G. Szalay, R. Ahlrichs, F.B. Brown, and J.G. 
Zhao, Int. J. Quantum Chem. 22, 149 (1988).

[39] H. Lischka, R. Shepard, R.M. Pitzer, I. Shavitt, M. Dallos, Th. Müller, P.G. Szalay, M. Seth, G.S. Kedziora, S. Yabushita, and Z. Zhang, Phys. Chem. Chem. Phys. 3, 664 (2001).

[40] T. Helgaker, H.J.Aa. Jensen, P. Jørgensen, J. Olsen, K. Ruud, H. Ågren, T. Andersen, K.L. Bak, V. Bakken, O. Christiansen, P. Dahle, E.K. Dalskov, T. Enevoldsen, H. Heiberg, H. Hettema, D. Jonsson, S. Kirpekar, R. Kobayashi, H. Koch, K.V. Mikkelsen, P. Norman, M.J. Packer, T. Saue, P.R. Taylor, and O. Vahtras, computer code DALTON, Release 1.0 (1997).

[41] T.H. Dunning, Jr., J. Chem. Phys. 90, 1007 (1989).

[42] R.A. Kendall, T.H. Dunning, Jr., and R.J. Harrison, J. Chem. Phys. 96, 6796 (1992).

[43] The potential-energy curves have been calculated using the MR-CISD method with Davidson correction. For Fig. 4(a) we have used an embedded cluster of $\mathrm{Li}_{25}{ }^{+} \mathrm{F}_{9}{ }^{-}$[see Fig. 5(f)] giving rise to nine different potential-energy curves of $A_{1}$ symmetry. For panel (b) we have used a $\mathrm{Li}_{20}{ }^{+} \mathrm{F}_{8}{ }^{-}$cluster (eight curves of $A_{1}$ symmetry). Panel (c) with nine curves of $A_{1}$ symmetry corresponds to Fig. 11(c) using an embedded $\mathrm{Li}_{26}{ }^{+} \mathrm{F}_{9}{ }^{-}$cluster for the representation of the surface. The zero of energy corresponds to the energy of the ionic state (positive projectile plus neutral surface) at large distance.

[44] W.J. Stevens, H. Basch, and M. Krauss, J. Chem. Phys. 81, 6026 (1984).

[45] N. Govind, Y.A. Wang, and E.A. Carter, J. Chem. Phys. 110, 7677 (1999).

[46] For these calculations we used the program package TURBOMOLE [47] which allows for a sufficiently large number of atoms and basis functions. The largest cluster corresponds to a cube of $5 \times 5 \times 5$ of $\mathrm{Li}^{+}$and $\mathrm{F}^{-}$ions with a $\mathrm{F}^{-}$in the center. This cluster is embedded in point charges such that the total size of the system is a $15 \times 15 \times 15$ ions. The positive point charges at the interface to the active cluster are replaced by active $\mathrm{Li}^{+}$ions in order to ensure that all $\mathrm{F}^{-}$a properly "surrounding" by active ions on all sides. (If this is done, the electron density at the boundaries of the active cluster is artificially distorted towards the point charges due to the missing "Pauli repulsion" by orbitals from nearest neighbors.) This leads to a total active cluster of $\mathrm{Li}_{146}{ }^{+} \mathrm{F}_{63}{ }^{-}$.

[47] R. Ahlrichs et al., computer code tURBOmOLE, Version 4 (1997).

[48] For the cluster ions we use the same basis set as for the calculation of the ionization potentials of the isolated clusters in Sec. III, i.e., the pseudobasis by Stevens, Basch, and Krauss [44] with truncation of the $p$-basis functions at the lithium ions. For $\mathrm{Na}^{+}$and $\mathrm{S}^{+}$we also use the pseudobasis [44] (without truncation of the $p$ functions). For $\mathrm{C}^{+}$and $\mathrm{H}^{+}$, we use the cc-pVDZ basis of Ref. [41].

[49] The identification of the ionic and covalent states is made through the dominant reference configuration in the MR-CI wave function.

[50] A.I. Boldyrev, N. Gonzales, and J. Simons, J. Phys. Chem. 98, 9931 (1994).

[51] J. Burgdörfer and E. Kupfer, Phys. Rev. Lett. 57, 2649 (1986).

[52] Q. Yan, J. Burgdörfer, and F.W. Meyer, Phys. Rev. B 56, 1589 (1997).

[53] F.W. Meyer, Q. Yan, P. Zeijlmans van Emmichoven, I.G. Hughes, and G. Spierings, Nucl. Instrum. Methods Phys. Res. B 125, 138 (1997).

[54] The diabatic curve is approximately constructed from the electron occupation of the different CI configurations. At an avoided crossing, two or several states have strong admixtures of the ionic reference configuration. The diabatic curve of the ionic state connects the two lines that have dominant ionic character on the right/left side of the avoided crossing, respectively.

[55] Handbook of Chemistry and Physics, edited by D.R. Lide (CRC Press, Boca Raton, 1995). 